\title{
Fitofotodermatite: um diagnóstico a recordar - Relato de caso
}

Ana Luísa Teixeira, ${ }^{1}$ Vera Teixeira ${ }^{2}$

\section{RESUMO}

Introdução: A fitofotodermatite consiste numa reação inflamatória causada pela exposição da pele a agente tópico vegetal fotossensibilizante contendo furocumarinas, seguida de exposição a radiação ultravioleta. O caso clínico apresentado mostra a importância de uma cuidada anamnese aquando da avaliação clínica, já que a mesma é considerada a pedra basilar para o diagnóstico desta patologia.

Descrição do caso: Homem, 59 anos, técnico de análises clínicas, com antecedentes pessoais de hipertensão arterial essencial medicada e controlada com ramipril 2,5mg e fototipo de pele Fitzpatrick II. Recorre à consulta por quadro de eritema linear e vesículas discretas de conteúdo claro na região anterior de ambos os antebraços que notou subitamente de manhã, na semana anterior. Negava prurido, mas referia sensação de calor e ardor nas lesões. Negava ainda conhecimento de fator desencadeante do quadro clínico, alergias conhecidas, aplicação de algum novo produto cosmético ou fármaco ou exposição solar prolongada, reforçando não ter havido alteração de hábitos diários. Após anamnese mais detalhada, o utente referiu que carregou em braços resíduos provenientes da poda do crescimento excessivo do seu terreno de cultivo, o que incluía arruda. O diagnóstico de fitofotodermatite foi assim estabelecido.

Comentário: A família das Rutaceae, onde está incluída a arruda, é a segunda família de plantas que tem mais casos associados de fitofotodermatite. O diagnóstico desta patologia é clínico, sendo a anamnese fundamental para apurar a exposição a plantas que contenham furocumarinas. Neste caso, a apresentação característica de eritema linear e vesículas, com sensação de ardor, sem prurido, aliada a história de exposição a planta produtora de furocumarinas permite estabelecer o diagnóstico. A prevenção é o melhor tratamento, já que a eficácia da aplicação de corticoides tópicos, anti-histamínicos e outras intervenções tem evidência limitada. A hiperpigmentação surge uma a duas semanas após a exposição e pode persistir por mais de um ano.

Palavras-chave: Dermatologia; Dermatite fototóxica; Relato de caso.

\section{INTRODUÇÃO}

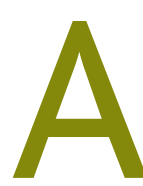

fitofotodermatite, também referida como fitofotodermatose, consiste numa reação inflamatória causada pela exposição da pele a um agente tópico vegetal fotossensibilizante contendo furocumarinas, seguida de exposição a radiação ultravioleta. ${ }^{1} \mathrm{O}$ seu diagnóstico é clínico e estabelecido mediante a apresentação característica das lesões dermatológicas e história de exposição aos agentes causais. Deste modo, não carece de exames complementares de diagnóstico, sendo a anamnese fundamental para apu-

1. Médica Interna de Medicina Geral e Familiar. USF O Basto, ACeS Alto Ave. Cabeceiras de Basto, Portugal.

2. Médica Assistente de Dermatovenereologia. Hospital de Santa Luzia, ULSAM.Viana do Castelo, Portugal. rar a exposição a plantas que contenham furocumarinas. ${ }^{2} \mathrm{~A}$ apresentação clínica consiste no aparecimento de um eritema linear (com ou sem vesículas) e hiperpigmentação pós-inflamatória. Não existe uma reação imunológica envolvida na sua fisiopatologia, pelo que não há necessidade de uma sensibilização prévia ao produto vegetal, podendo qualquer indivíduo ser afetado. ${ }^{1}$

Pela exposição a ambos os fatores necessários para o desenvolvimento da fitofotodermatite, esta patologia é frequente em trabalhadores agrícolas ou jardineiros que trabalham ao ar livre.

O caso clínico apresentado demonstra a importância de uma cuidada anamnese aquando da avaliação clínica, já que a mesma é considerada a pedra basilar para o diagnóstico correto desta patologia. 


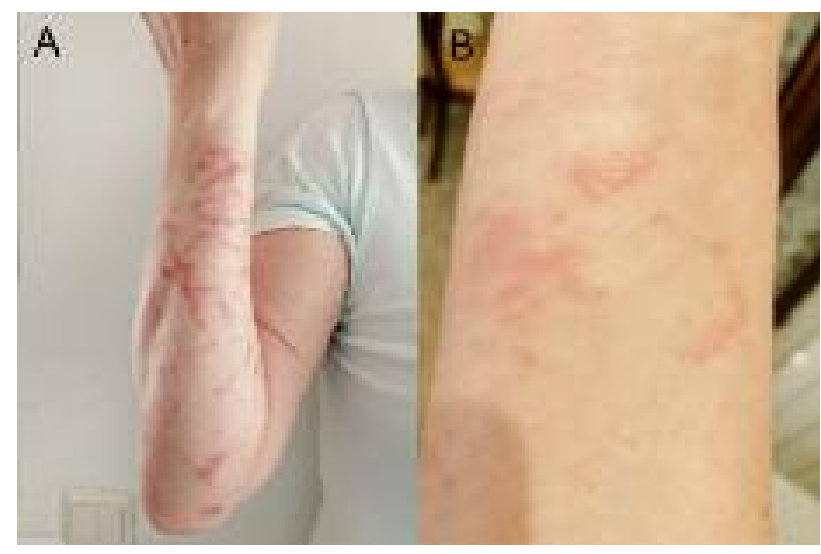

Figura 1. A. Lesões apresentadas na consulta. A região do pulso protegida pelas luvas não apresenta lesões; B. Fotografia do doente com algumas vesículas intactas.

Nota: Fotografias obtidas com consentimento escrito do utente.

\section{DESCRIÇÃO DO CASO}

Homem, 59 anos de idade, técnico de análises clínicas. Antecedentes pessoais: hipertensão arterial essencial medicada e controlada com ramipril 2,5mg; fototipo de pele Fitzpatrick II.

História da doença atual: o utente recorre à consulta aberta da sua unidade de saúde em maio de 2020 por aparecimento de quadro de eritema linear e vesículas discretas de conteúdo claro (não íntegras na consulta, mas com fotografia do doente para confirmação) na região anterior de ambos os antebraços, que havia notado subitamente de manhã, cerca de uma semana antes. Negava prurido, mas referia sensação de calor e ardor nas lesões. Negava conhecimento de fator desencadeante do quadro clínico, alergias conhecidas, aplicação de algum novo produto cosmético ou fármaco ou exposição solar prolongada, reforçando não ter existido qualquer alteração dos hábitos diários. Negava igualmente antecedentes de quadro clínico semelhante no passado, bem como outro tipo de reações de fotossensibilidade induzida.

Após realização de anamnese cuidada, o utente referiu que na semana anterior à consulta, enquanto trabalhava no campo, carregou nos braços expostos todos os resíduos provenientes da poda do crescimento excessivo do seu terreno, o que incluía a planta arruda, utilizada para afastar pragas na agricultura biológica. As mãos do utente estariam protegidas por luvas; no en-

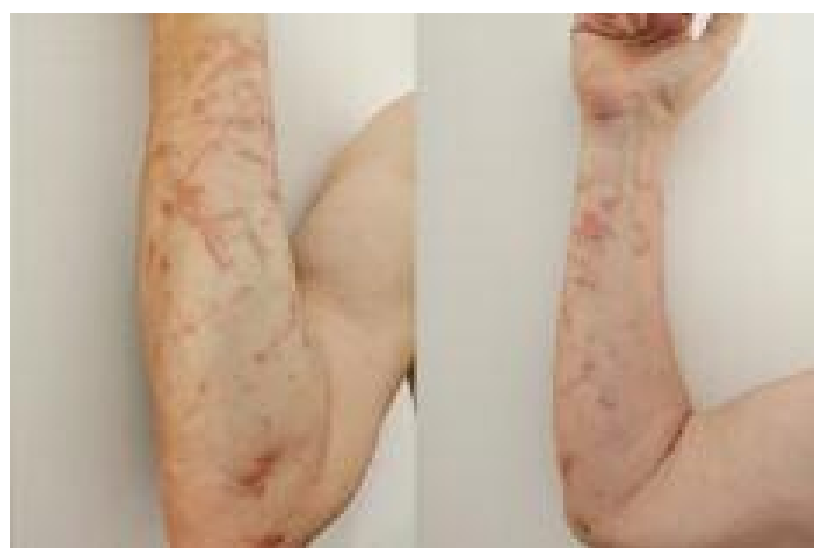

Figura 2. Evolução das lesões duas semanas após a avaliação inicial. Nota: Fotografias obtidas com consentimento escrito do utente.

tanto, a $t$-shirt de mangas curtas que vestia deixava os braços expostos ao sol. A Figura 1 mostra as lesões apresentadas pelo utente na consulta.

O diagnóstico de fitofotodermatite foi estabelecido e iniciou-se aplicação tópica de valerato de dexametasona $1 \mathrm{mg} / \mathrm{g}$ duas vezes por dia, durante cinco dias. Foram agendadas reavaliações às duas semanas e um mês após a consulta inicial. A Figura 2 mostra a evolução das lesões duas semanas após a avaliação.

Um mês após a primeira consulta constatou-se a presença de hiperpigmentação nas regiões previamente eritematosas, achado típico dos quadros de fitofotodermatite (Figura 3).

\section{COMENTÁRIO}

A fitofotodermatite está incluída no grupo das reações dermatológicas fototóxicas. ${ }^{3}$ A fototoxicidade é uma reação cutânea não alérgica induzida por agentes tópicos (como é o caso na fitofotodermatite) ou sistémicos (e.g., fármacos administrados por via oral). A fototoxicidade ocorre quando o agente absorvido pela pele em concentrações apropriadas é exposto a quantidades adequadas de luz com um determinado comprimento de onda, geralmente raios UVA. Teoricamente, esta é uma reação que não necessita de sensibilização e que deveria ocorrer em todos os indivíduos expostos aos agentes/radiação responsáveis. No entanto, na prática clínica a resposta é variável. ${ }^{3}$ A fitofotodermatite é um diagnóstico clínico estabelecido mediante apresentação característica das lesões e história de 


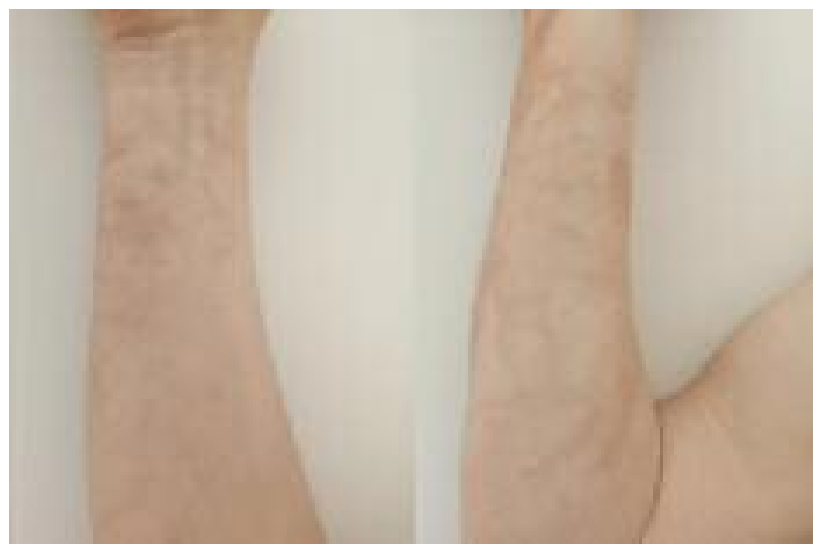

Figura 3. Estado das lesões com um mês de evolução. A hiperpigmentação substituiu o eritema.

Nota: Fotografias obtidas com consentimento escrito do utente.

exposição aos agentes causais, não carecendo de meios complementares de diagnóstico. Assim, a anamnese é fundamental. ${ }^{2}$

Na fitofotodermatite as substâncias presentes nas plantas capazes de provocar estas reações são as furocumarinas. Duas grandes famílias de plantas são as principais causadoras desta patologia: Apiaceae e $R u$ taceae (que inclui frutos cítricos). Alguns membros da família das amoreiras (Moraceae) ou das ervilhas ( $\mathrm{Fa}$ baceae) também contêm furocumarinas. ${ }^{1}$

Dentro da família de plantas das Apiacea podem ser destacadas a Ammi majus, comummente conhecida como âmio-maior ou âmio-vulgar; Angelica archangelica e Angelica sylvestris, comummente conhecidas como angélica e angélica-silvestre ou erva-piolheira, respetivamente; o aipo (Apium graveolens); o funcho (Foeniculum vulgare); a pastinaca ou cherovia (Pastinaca sativa), cultivada na zona da Serra da Estrela e Covilhã; e, por fim, a salsa (Petroselinum crispum). Na família das Rutaceae, algumas das plantas e frutos responsáveis pela fitofotodermatite são o limão (Citrus limon), a lima (Citrus aurantiifolia), a toranja (Citrus paradisii), a arruda (Ruta graveolens) e várias espécies de laranjas (Citrus sinensis, Citrus aurantium, Citrus bergamia), incluindo a bergamota, presente em alguns perfumes. Na família das Moraceae destaca-se a figueira (Ficus carica), particularmente os seus rebentos e folhas. Por oposição, os frutos da figueira não apresentam concentrações elevadas destes compostos. ${ }^{1,3}$
A família das Apiaceae é, de todas, aquela que tem mais casos de fitofotodermatite associados, seguida pela família das Rutaceae ${ }^{1,3}$ A arruda, o agente causal deste caso clínico, foi historicamente utilizada como tratamento caseiro de urticária, verrugas e erisipela. Extratos de arruda têm também sido utilizados como repelente de insetos e há casos descritos de fitofotodermatite após aplicação corporal de infusões de arruda pelas suas propriedades «místicas e purificadoras». ${ }^{1,4}$ A arruda é a causa mais frequente de fitofotodermatite em jardins ingleses.

Estas reações são particularmente frequentes na Primavera e no Verão, quando a concentração de furocumarinas é maior neste tipo de plantas e os indivíduos apresentam maior percentagem de pele exposta à radiação solar. ${ }^{1,5} \mathrm{~A}$ apresentação clínica pode ser ligeira, caracterizando-se somente por eritema seguido de hiperpigmentação ou apresentar manifestações mais graves. As apresentações mais exuberantes caracterizam-se por eritema e parestesias num curto período de tempo após a exposição, seguido de edema, vesículas, bolhas e sensação de ardor ou queimadura 24 horas após a exposição. ${ }^{1,3} \mathrm{O}$ pico das manifestações acontece às 72 horas. ${ }^{1}$ As lesões são dolorosas, mas não pruriginosas. ${ }^{1}$ A apresentação do eritema e vesículas ou bolhas em padrão linear figurado (decorrentes dos locais onde a seiva dos ramos do agente entrou em contacto com a pele) somente em áreas expostas à luz solar confere-lhe um aspeto característico. A hiperpigmentação aparece uma a duas semanas após a exposição e pode persistir por mais de um ano. ${ }^{3}$

Os diagnósticos diferenciais desta patologia incluem dermatite de contacto, fototoxicidade relacionada com fármacos, queimaduras graves, erupção polimorfa à luz, herpes simplex, entre outros. Neste caso clínico particular, a fototoxicidade induzida por fármacos deveria ser considerada, uma vez que o doente estava medicado com ramipril, um inibidor da enzima de conversão da angiotensina, fármaco que pode ser responsável por este tipo de reações. ${ }^{2,5}$

A prevenção é o tratamento mais eficaz para a fitofotodermatite. ${ }^{1-2} \mathrm{~A}$ evicção da manipulação deste tipo de plantas com os membros expostos ao sol é essencial. Ainda assim, após a exposição, a lavagem precoce com água e sabão pode prevenir a reação. ${ }^{1} \mathrm{~A}$ eficácia de outro tipo de tratamentos tem evidência limitada. ${ }^{2}$ Em 
determinados casos podem ser prescritos corticoides tópicos de potência moderada, duas vezes por dia durante duas semanas, anti-histamínicos ou compressas frias para o alívio da dor ou prurido; no entanto, estas recomendações são baseadas em opiniões de peritos. ${ }^{2,4,6}$ Está recomendado follow-up às quatro semanas de evolução. ${ }^{6}$

Sendo a fitofotodermatite um diagnóstico clínico, uma anamnese detalhada é fundamental. ${ }^{2} \mathrm{Nem}$ sempre é fácil para o doente identificar o contacto, já que muitas vezes refere que as lesões apareceram subitamente. Por esse motivo, o médico de família deve ativamente investigar os dias que precederam o aparecimento das manifestações cutâneas. É fundamental a familiarização com o aspeto característico das lesões e com o diagnóstico para uma correta identificação do mesmo. Este caso clínico tem, assim, como objetivo recordar uma patologia que, apesar de frequente, pode passar despercebida.

\section{REFERÊNCIAS BIBLIOGRÁFICAS}

1. Bolognia JL, Jorizzo JJ, Schaffer JV, Callen JP, Cerroni L, Heymann WR, et al. Dermatology (Vol. I). 3rd ed. Elsevier Saunders; 2012.

2. Harshman J, Quan Y, Hsiang D. Phytophotodermatitis: rash with many faces. Can Fam Physician. 2017;63(12):938-40.

3. Habif TP. Clinical dermatology: a color guide to diagnosis and therapy. 5th ed. Mosby Elsevier; 2009. ISBN 9780723435419

4. Bravo BS, Balassiano LK. Fitofotodermatite por Ruta graveolens com manifestação cutânea extensa [Phytophotodermatitis due to Ruta graveolens with extensive cutaneous manifestation]. Rev Bras Queimaduras. 2014;13(3):177-9. Portuguese

5. Buxton PK, Morris-Jones R. ABC of dermatology. 5th ed. BMJ Books; 2009. ISBN 9781405170659

6. Weston WL, Lane AT, Morelli JG. Color textbook of pediatric dermatology. 4th ed. Edinburgh: Mosby; 2007. ISBN 9780323049092

\section{CONFLITO DE INTERESSES}

Os autores declaram não possuir quaisquer conflitos de interesse.

\section{ENDEREÇO PARA CORRESPONDÊNCIA}

Ana Luísa Teixeira

E-mail: aluisafteixeira@gmail.com

https://orcid.org/0000-0003-2706-5843

Recebido em 21-12-2020

Aceite para publicação em 10-05-2021

\section{ABSTRACT}

\section{PHYTOPHOTODERMATITIS: A DIAGNOSIS TO KEEP IN MIND - CASE REPORT}

Introduction: Phytophotodermatitis is an inflammatory reaction caused by skin exposure to a photosensitizing plant extract, followed by exposure to ultraviolet radiation. This case report highlights the importance of a well-thought anamnesis as part of the clinical evaluation, as it is the cornerstone for the correct identification of this diagnosis.

Case description: Male, 59 years old, clinical analysis technician with a history of essential hypertension, medicated and controlled with ramipril $2.5 \mathrm{mg}$, and Fitzpatrick II phototype. The patient came in with a manifestation of linear erythema and discrete vesicular lesions with clear content on the anterior forearms, which he noticed suddenly one morning, the week before. The patient denied itching but felt pain and stinging. He denied knowledge of any triggering factors, known allergies, new cosmetic products or drugs, prolonged sun exposure, and reinforced that there hadn't been any changes in his daily routine. After more detailed anamnesis the patient mentioned having carried on bare arms, under sunlight exposure, the pruning scraps of his biological agriculture vegetable garden, which included rue. Phytophotodermatitis was therefore diagnosed.

Commentary: The Rutaceae plant family, in which rue is included, is the second plant family with the most case reports of phytophotodermatitis. This is a clinical diagnosis and therefore the anamnesis is of central importance to identify exposure to potential furocoumarin-containing plants. In this case, the characteristic presentation of painful, non-pruritic, linear erythema and bullae, with the history of exposure to photosensitizing plant extract enabled the diagnosis. Prevention is the best treatment given the limited evidence on the efficacy of topical steroids, antihistamines, and other interventions. Skin hyperpigmentation presents within one or two weeks after exposure and can last for a year.

Keywords: Dermatology; Phototoxic dermatitis; Case reports. 